\title{
A self-paced BCI with a collaborative controller for highly reliable wheelchair driving: experimental tests with physically disabled individuals
}

\author{
Aniana Cruz, Member, IEEE, Gabriel Pires, Member, IEEE, Ana Lopes, Member, IEEE, Carlos Carona, \\ and Urbano J. Nunes Senior Member, IEEE
}

\begin{abstract}
Brain-controlled wheelchairs (BCWs) are a promising solution for people with severe motor disabilities, who cannot use conventional interfaces. However, the low reliability of electroencephalographic signal decoding and the high user's workload imposed by continuous control of a wheelchair requires effective approaches. In this paper, we propose a self-paced P300based brain-computer interface (BCI) combined with dynamic time-window commands and a collaborative-controller. The selfpaced approach allows users to switch between control and non-control states without requiring any additional task or mental strategy, while the dynamic time-window commands allow balancing the reliability and speed of the BCI. The collaborative controller, combining user's intentions and navigation information, offers the possibility to navigate in complex environments and to improve the overall system reliability. The feasibility of the proposed approach and the impact of each system component (self-paced, dynamic time-window and collaborative controller) were systematically validated in a set of experiments conducted with seven able-bodied participants and 6 physically disabled participants steering a robotic wheelchair in real office-like environments. These 2 groups controlled the BCW with a final driving accuracy greater than $99 \%$. Quantitative and subjective results, assessed through questionnaires, attest to the effectiveness of the proposed approach. Altogether, these findings contribute to improve the usability of $\mathrm{BCWs}$ and hence the potential for their use by target users in home settings.
\end{abstract}

Index Terms-Brain-computer interface (BCI), self-paced, dynamic time-window, collaborative control, robotic wheelchair, physically disabled, quantitative and subjective assessment.

\section{INTRODUCTION}

$\mathbf{P}$ EOPLE suffering from conditions that affect neuromuscular structures and functions tend to lose a significant degree of autonomy in daily living activities. Powered

This work has been financially supported by Portuguese foundation for science and technology (FCT) under grant B-RELIABLE (PTDC/EEIAUT/30935/2017) and grant ISR-UC UID/EEA/00048/2019. Aniana Cruz was supported by the Ph.D. FCT Scholarship SFRH/BD/111473/2015.

Aniana Cruz is with University of Coimbra, Institute of Systems and Robotics, Portugal, anianabritodisr.uc.pt

Gabriel Pires is with the Institute of Systems and Robotics, University of Coimbra, Portugal, and also with the Polytechnic Institute of Tomar, Portugal gpireseisr.uc.pt

Ana Lopes is with the Institute of Systems and Robotics, University of Coimbra, Portugal, and also with the Polytechnic Institute of Tomar, Portugal anacriseisr.uc.pt

Carlos Carona is with University of Coimbra, Center for Research in Neuropsych. and Cognitive-Behavioral Interv. and also with the Cerebral Palsy Association of Coimbra, Portugal ccarona@fpce.uc.pt

Urbano J. Nunes is with University of Coimbra, Institute of Systems and Robotics, Department of Electrical and Computer Engineering, Portugal urbanoddeec.uc.pt wheelchairs may help them to increase their levels of mobility and quality of life [1]. However, many of them become unable to use conventional interfaces, as a result of impairment severity or physical ability deterioration [2]. For those with severe motor impairments, brain-computer interfaces (BCIs) may be an alternative solution as it is possible to send commands through brain signals without requiring muscle activity [3], [4], [5], [6]. Yet, using a BCI to control a robotic wheelchair is a very challenging task because $\mathrm{BCI}$ has low transfer rates and limited accuracy [7]. Controlling a BCI system requires continuous and high levels of attention and focus, which imposes a high mental and physical workload that limits its usability. In turn, this workload can cause attention shifts and fatigue, resulting in even greater uncertainty in decoded brain commands. In the context of brain-controlled wheelchairs (BCWs) steered in real-world scenarios, the low reliability and rate of BCI commands can lead to disastrous safety consequences for the user and the system [8]. For this reason, when compared to other BCI applications such as spellers or games, $\mathrm{BCWs}$ require much higher reliability and general usability, which is only possible if they integrate an assistive navigation system (ANS) that perceives the wheelchair's surroundings and performs suitable and smooth trajectories, considering the user intents. This can be accomplished by combining user and machine outputs in a so-called collaborative controller [9], [5], [10], [6], [11], allowing BCI commands, which encode highlevel goals, to be provided at sparse intervals without the need for precise, low-level continuous steering.

The aforementioned collaborative approach may not yet be sufficient for effective use of a BCI because the user still needs to provide BCI commands in regular time-windows and has to be continuously focused, which is a mentally demanding task [12], [13], [10]. Self-paced control (also known as asynchronous control) provides the possibility for users to send BCI commands only when they wish to, at their own pace. This is therefore a very desirable feature, which can lead to less mental effort and more natural driving interaction [14], [5], [15], [6], [16], [17], [18]. To implement a self-paced $\mathrm{BCI}$, the system must automatically recognize control and noncontrol states. In a state of non-control, it is understood that the user does not want to select any command.

Brain-controlled wheelchairs have been researched for more than one decade. They are mainly based on three neural mechanisms: motor imagery (MI) [5], [16], [18], steadystate visual evoked potential (SSVEP) [13], [10], [19], [11], 
and P300 event-related potential (ERP) [12], [20], [6], [21]. Hybrid brain-actuated wheelchairs have also been proposed combining different neural mechanisms or combining brain signals with other physiological signals [14], [15], [22], [23], [17]. They may be used to increase the system reliability or to adapt to users functionality. For example, in [15] it is proposed the use of MI for selecting left, right, forward and backward commands, P300 and MI for acceleration or deceleration, and eye-blinking to issue stop commands. In [17], the asynchronous control is achieved based on a leftright motor imagery sequence and commands are selected through a P300-based interface. Most proposed BCI systems consider a fixed time interval to select the desired command, meaning that a fixed number of stimuli sequences (P300based BCIs) or a fixed time-window (MI and SSVEP based BCIs) are required for decoding the user's intention [5], [22], [13], [10], [19], [6], [21]. The use of a dynamic time-window to issue $\mathrm{BCI}$ commands is also a desirable feature, as the speed of the system can be adjusted online to the user's performance, thereby increasing BCI accuracy, however very few have used this approach [20]. BCWs can use either highlevel commands [22], [19], [6], [14], [16], [21], [23] or lowlevel commands [5], [13], [20], [10], [15]. The use of highlevel commands requires the robot to be able to perform autonomously safe and effective navigation without user's aid (commands can be either global, such as 'kitchen', 'wc', or local, such as 'door', 'go-left'). With low-level commands, the user can steer the wheelchair with raw commands (e.g., 'forward', 'left', 'increase speed'). Although this approach is flexible, as the user can control any specific motion, it is highly demanding and almost impossible to use in real-world environments, even with a collaborative controller. So far, the reported experimental tests combining user's intent and context awareness in a collaborative controller have been conducted in very structured environments [14], [13], [4], [10], [19], [23], [11] or open spaces [22], and in semi-structured environments [12], [5], [15], [6], [16]. Moreover, just a few works report experiments conducted with motor impaired participants [21], [23]. For more extensive surveys comparing different BCWs approaches, please refer to [24] and [8].

The main goal of this work is to research ways to increase both the reliability and usability of BCWs, extending our previous work [6], which was focused on the robotic navigation system, and reported preliminary data of a selfpaced BCI approach. In the current study, a new set of experiments was carried out including participants with severe motor disabilities. We propose a P300-based BCW that combines the previously developed collaborative controller with self-paced control and a new dynamic time command approach. The impact of each of the three aforementioned control modes on three dimensions of usability (reliability, workload, and naturalness) of the overall system is assessed through systematic tests. Our robotic platform - RobChair - was ergonomically adapted to be used by severely motor impaired participants. Several navigation tasks were carried out in real office-environment by a group of 6 individuals with severe motor disabilities and by a control group of 7 ablebodied participants. The effectiveness of the proposed methods and approaches were assessed based on quantitative metrics, as well as on subjective questionnaires to asses user experience. The main contributions of the current study are: 1) proposal and validation of a new dynamic time-window approach for BCI commands based on the degree of the classifier's confidence, and its combination with the self-paced approach, that adjusts the BCI speed to the user's performance over time. To the best of our knowledge, very few works have used dynamic time-window methods in brain-actuated wheelchairs (and those used different approaches) and none have done so in a non-simulated environment [20]. This automatic adjustment decreases the users' performance fluctuations that may arise from changes in the users' attention, thus maintaining the most stable reliability naturally; 2) validation of a self-paced approach that frees the user from being continuously focused on the BCI. This is achieved through a non-control state that does not involve any additional task for the user, as he/she only has to be relaxed in a state of inattention. At the same time, the approach also tunes the rate of false positives, which is different from other P300-based brain-actuated wheelchair approaches. The proposed self-paced detector contributes to a natural BCI operation increasing the usability of the system; 3) combination of three impactful features in a single framework: self-paced control, dynamic adjustment of timewindow commands and collaborative control, aiming at high overall reliability. This led to an overall performance greater than $99 \%$ without decreasing the BCI speed, which shows the feasibility of the approach in this application but which can be extended to other different contexts (e.g., predictive spellers); 4) validation of the $\mathrm{BCW}$ in a realistic office-like environment with severe physically disabled participants. This represents a contribution to the effective validation of BCI approaches, as most studies have validated their approaches only with healthy participants.

Both quantitative and subjective results clearly support the importance of the proposed overall solution. This work represents an important effort in improving and assessing the usability of BCWs, moving toward its potential use by target users.

\section{EXPERIMENTAL DESIGN}

\section{A. Participants}

This study comprises two groups of participants: 7 ablebodied users, referred to as Group I, and 6 participants with severe motor disabilities referred to as Group II (see Tables I and II). The study was ethically assessed and approved by the board of the Cerebral Palsy Association of Coimbra (APCC) and was conducted complying with the code of Ethics of the Declaration of Helsinki. Informed consent was obtained from all participants, explaining the aims of the study, their role as participants (e.g., voluntary participation) and the ethical commitments of the research team (e.g., data anonymization, guarantee of confidentiality). The sample of able-bodied users (S1-S7) was composed of students and researchers with ages between 21 and 32 years old, with a mean age of 23.7 years. Only one participant had previous experience with P300-based $\mathrm{BCI}$ and none had experience in driving a wheelchair. Table I 


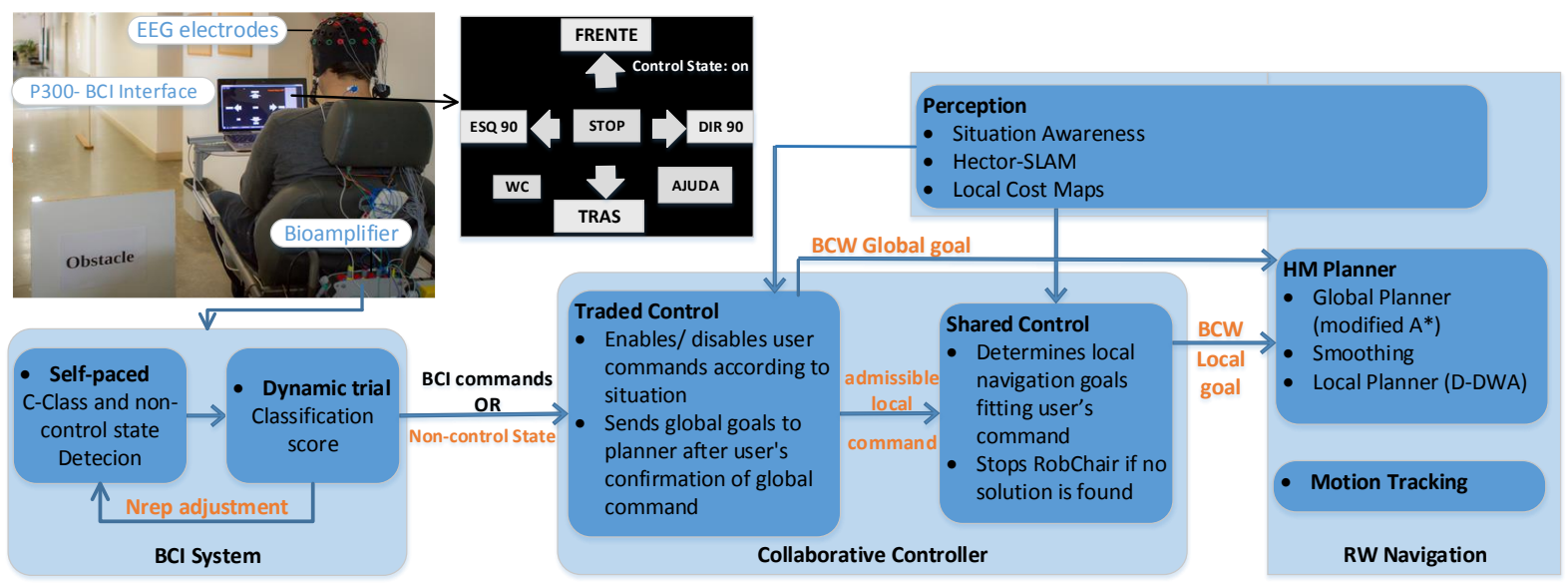

Fig. 1: Schematic representation of the overall BCW system, which is composed of three main modules: BCI System, Collaborative Controller, and Robotic Wheelchair Navigation.

TABLE I: Able-bodied participants

\begin{tabular}{llllllll}
\hline Subjects & S1 & S2 & S3 & S4 & S5 & S6 & S7 \\
\hline Age & 32 & 25 & 22 & 22 & 21 & 21 & 23 \\
Sex & F & F & M & M & M & M & M \\
BCI experience & YES & NO & NO & NO & NO & NO & NO \\
\hline
\end{tabular}

presents their ages, gender and their previous BCI experience. Group II (P1-P6) included outpatients from APCC with ages between 21 and 50 years old, averaging 37.5 years old. Primary clinical diagnoses for the latter group included cerebral palsy ( 2 cases), spinal cord injury ( 1 case), agenesis of the four members (1 case), limb-girdle muscular dystrophy (1 case) and Duchenne muscular dystrophy (1 case). Table II contains a more detailed description of the disabled participants, as well as a summary of their clinical and functional data. They were all dependent on human assistance in daily activities but still able to use powered wheelchairs independently with customized interfaces (even though some of them with great difficulty).

\section{B. BCI Graphical User Interface and Commands}

The BCI uses a visual oddball paradigm to evoke P300 event-related potentials. The visual paradigm comprises the following 7 steering commands: 'FORWARD', 'BACK', 'LEFT90', 'RIGHT90', 'STOP', 'WC', and 'HELP', in Portuguese ('FRENTE', 'TRAS', 'ESQ90', 'DIR90', 'STOP', 'WC', 'AJUDA', respectively), as depicted in Fig. 1. These symbols flash randomly with an highlight time of $100 \mathrm{~ms}$ and an inter-stimulus interval of $75 \mathrm{~ms}$, resulting in a stimuli onset asynchrony (SOA) of $175 \mathrm{~ms}$. For every round of the oddball paradigm each symbol is flashed once. An EEG data segment (epoch) with 256 time samples (one second) is extracted for each stimulus onset. Because of the low signal-to-noise ratio of P300 ERPs, several rounds are usually required to collect several target epochs, to improve the target classification.

For the conventional approach (here called as static trial) the number of repetitions/rounds $\left(\mathbf{N}_{r e p}\right)$ necessary to select a target is fixed, settled according to the performance of users in the calibration phase. For the dynamic trial approach (dynamic-time commands), the number of repetitions per trial during the online operation varies according to the target classification score. The overall trial time (TT) needed for symbol classification is computed from

$$
T T=N_{\text {rep }} \times N_{s} \times S O A+C T
$$

where $N_{s}=7$ is the number of symbols, and $C T=1$ is the time associated with the last flash of the trial. The Inter-Trial Interval (ITI), i.e. the time between each set of rounds, was set to one second.

\section{Calibration sessions}

Before starting the driving tasks, each participant performed a calibration session to obtain the classification models. Participants were seated in the RobChair with the computer screen positioned in front of them at a distance of approximately $30 \mathrm{~cm}$, in the same conditions they have while driving the wheelchair. Participants were instructed to focus on the predefined target commands, successively provided at the top of the screen, and to mentally count whenever a target command flashes. Calibration consisted of a sequence of 9 symbols, and 9 rounds per symbol, collecting 81 target epochs and 486 nontarget epochs, taking about 2 minutes. Participants performed only one calibration, from which all classification models and parameters were obtained to control the 3 performed tasks.

\section{RobChair system}

RobChair is a robotic wheelchair (RW) with differential actuation, equipped with optical encoders coupled to each motorized wheel and an hokuyo UTM-30LX scan laser. Its navigation architecture is implemented in ROS and is composed of three main modules: perception, planning, and motion tracking. Currently, the perception module is composed of: situation awareness; Simultaneous Localization and Mapping (SLAM), performed with Hector-SLAM [25], and multiresolution local cost maps, as described in [6]. The planning module is based on the hybrid motion (HM) planner, which is 
TABLE II: Motor disabled participants

\begin{tabular}{|c|c|c|c|c|c|c|c|}
\hline Subjects & Age & Gender & $\begin{array}{l}\text { BCI ex- } \\
\text { perience }\end{array}$ & Diagnosis & $\begin{array}{l}\text { Type of dis- } \\
\text { ability }\end{array}$ & Level of motor functionality & $\begin{array}{l}\text { Interface used to steer } \\
\text { powered wheelchair }\end{array}$ \\
\hline $\mathrm{P} 2$ & 35 & $\mathrm{~F}$ & NO & $\begin{array}{l}\text { Agenesis of the four } \\
\text { members (and sen- } \\
\text { sory impairment) }\end{array}$ & congenital & $\begin{array}{l}\text { Moderate autonomy in day-to-day ac- } \\
\text { tivities. Very small limbs; Slight move- } \\
\text { ment disturbance. }\end{array}$ & $\begin{array}{l}\text { Joystick controlled by } \\
\text { stump; Good efficiency. }\end{array}$ \\
\hline P4 & 45 & M & NO & $\begin{array}{l}\text { SCI (and sensory im- } \\
\text { pairment) }\end{array}$ & acquired & $\begin{array}{l}\text { Head and upper limbs con- } \\
\text { trol(tetraparesis). Moderate autonomy } \\
\text { in day-to-day activities. }\end{array}$ & $\begin{array}{l}\text { Joystick controlled by } \\
\text { hand; Good efficiency. }\end{array}$ \\
\hline P5 & 25 & M & NO & $\begin{array}{l}\text { Limb-girdle muscular } \\
\text { dystrophy }\end{array}$ & congenital & $\begin{array}{l}\text { Low autonomy in day-to-day activities. } \\
\text { Proximal muscle weakness. }\end{array}$ & $\begin{array}{l}\text { Joystick controlled by } \\
\text { hand; Good efficiency. }\end{array}$ \\
\hline P6 & 21 & M & NO & $\begin{array}{l}\text { Duchenne muscular } \\
\text { dystrophy }\end{array}$ & congenital & $\begin{array}{l}\text { Low autonomy in day-to-day activities. } \\
\text { Muscle weakness. }\end{array}$ & $\begin{array}{l}\text { Joystick controlled by } \\
\text { hand; Good efficiency. }\end{array}$ \\
\hline
\end{tabular}

composed of a global planner based on a modified version of the $\mathrm{A}^{*}$ algorithm, a smoother and a Double-Dynamic Window (D-DWA) approach for local planning. The collaborative controller is a decision-making module composed of two layers: traded and shared controllers (see Fig. 1). It receives sparsely issued high-level commands from the P300-based BCI that can either be global or local commands. Global commands consist of a set of target goals belonging to the navigational space (e.g. WC). As soon as a user selects a global command through the P300-based BCI, the traded controller is in charge of validating the command after user confirmation, sending it directly to the global planner. On the other hand, local commands allow the RW to navigate between local goals previously defined on the topological map (also referred to as decision points). A decision point is defined as an ambiguous place in the map because it allows several directions to be taken from there (e.g. intersections or bifurcations). In these situations, the user provides a direction through the P300-based BCI (e.g., FORWARD, BACK, etc.), and the collaborative controller determines the closest local goal in the direction provided by the user. Although these commands might be understood as low-level, they are, in fact, high-level commands because they provide a way to choose a pre-defined local goal. If the user issues a local command the traded controller enables/disables that command depending on the information provided by the situation awareness module (e.g. if a user issues the LEFT command but it is only possible to move forward, the command is disabled). The shared controller is in charge of determining the appropriate local navigation goal according to the admissible local command provided by the traded controller, situation, place being navigated, and topological map information (i.e. predefined subgoals or decision points). More details on the collaborative control algorithm can be found in [6]. RobChair was ergonomically adapted with help of APCC staff, to be used by severe motor impaired participants.

\section{E. Navigation scenarios}

The experiments consisted in steering the RobChair in a real indoor office environment. Participants performed three navi- gation tasks as described below. The first task used the selfpaced BCI with static trial time (STT), the second one used the self-paced BCI with dynamic trial time (DTT), and the third one used a non self-paced approach. The order of the tasks was the same for all participants, Task1-Task2 for the physically disabled participants and Task1-Task2-Task3 for the ablebodied group. Each participant performed the experiments on the same day. Before starting the task, each participant went through the designated path seated in the wheelchair, while an external operator was driving the wheelchair using a joystick, and the decision points were shown. Then, at the starting point of the route, after the calibration, each participant was enabled to become familiar with the interface, selecting commands, but with the wheelchair stopped. The familiarization time was variable between participants, ensuring that each one understood the task.

Task1 - Collaborative and self-paced control with STT. Users steered the RobChair following the map route depicted in Fig. 2, from an office, represented as START, to a lab, represented as END using the self-paced P300-based BCI with STT, whose implementation is explained in section III-B. Before starting the tests, participants were instructed about the route, which included three narrow doorways $(\mathrm{B}, \mathrm{I}$, and $\mathrm{K})$, two small obstacles (D and F) and two large obstacles ( $E$ and $G)$. The minimum number of decisions to reach the final destination was 5 , i.e. participants had to provide commands at each decision point (A, C, H, J, and L). However, the $\mathrm{BCI}$ is always outputting a command (target symbol or non-control state) at every trial. To detect the occurrence of false positives and false negatives, users were instructed to press an adapted switch whenever the BCI system selected an erroneous command, and someone was always behind for double checking.

Task2 - Collaborative and self-paced control with DTT: The navigation task consisted of moving from the lab signed by START to the hall near the ELEVATOR, as shown on the map in Fig. 3, using the self-paced P300-based BCI with the DTT approach. This route included a room, passage through two doors and navigation in a corridor. To perform this 


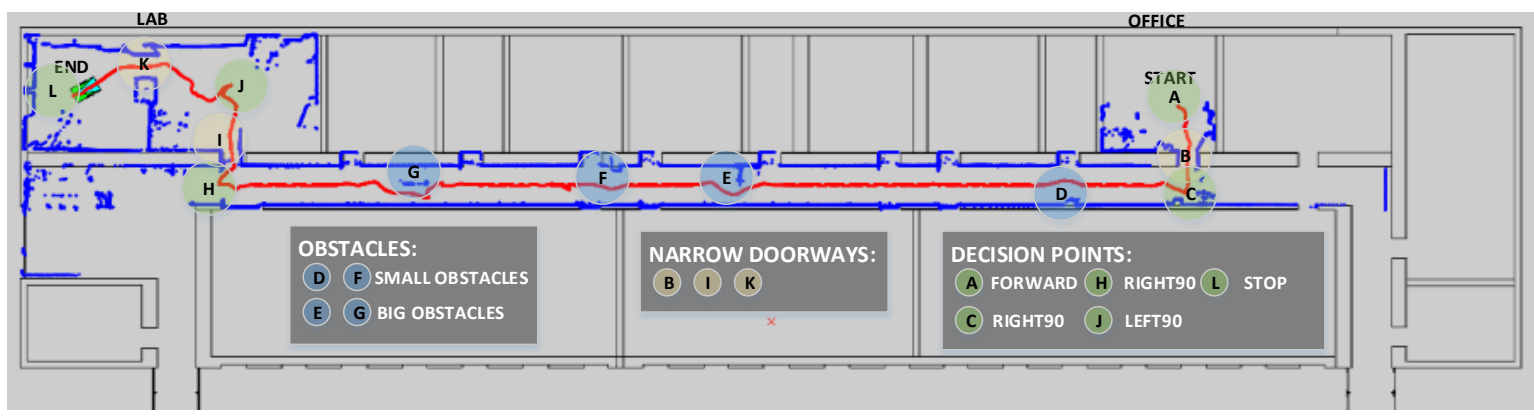

Fig. 2: Map with scenarios in which the participants performed Task1 and Task3 with 4 obstacles (D, F, E and G), 3 narrow passages (B, I and $\mathrm{K})$ and 5 decision points (A, C, H, J and L).

navigation task, users were required to provide commands to start it and to choose the appropriate local goal in decision points (A, C, E, F, and $\mathrm{H})$. As in STT, the BCI is always outputting a command (target symbol or non-control state) at every dynamic trial.

Task3 - Collaborative and non self-paced control with STT: In this task, we have evaluated the non-self-paced control in which the user had to provide a target selection at every trial, as in this mode the BCI could not detect the non-control state. The route was the same as in Task 1 , which consisted of going from OFFICE to LAB. RobChair's speed was programmed to slow down at every decision point, that is, points in which the robot could not make a decision without an appropriate user command.

\section{F. EEG Data Acquisition and Preprocessing}

EEG was recorded with a 16-channel g.USBamp bioamplifier at positions $\mathrm{Fz}, \mathrm{Cz}, \mathrm{C} 3, \mathrm{C} 4, \mathrm{CPz}, \mathrm{Pz}, \mathrm{P} 3, \mathrm{P} 4, \mathrm{PO} 7, \mathrm{PO}$, $\mathrm{POz}$ and $\mathrm{Oz}$ according to the international extended 10-20 standard system (see a photo of the system setup in Fig. 1). The reference electrode was placed at right or left earlobe and the ground at AFz. The EEG signals were acquired with active $\mathrm{Ag} / \mathrm{AgCl}$ electrodes, sampled at $256 \mathrm{~Hz}$, and filtered using a band-pass filter between 0.5 and $30 \mathrm{~Hz}$ and notch-filtered at $50 \mathrm{~Hz}$.

\section{Methods: Self-PaCed P300-BASEd BCI AND DYNAMIC TRIAL}

\section{A. Online Classification Pipeline}

The online classification pipeline of the self-paced P300 BCI system is schematically represented in Fig. 4. After preprocessing, the data is segmented into epochs of 1 second, and then the epochs are normalized to zero mean and unitary standard deviation. The number of repetitions per trial is selected from the calibration session. Then, the normalized epochs of the $N_{r e p}$ repetitions are averaged for each channel and a feature extractor is applied, namely a statistical spatial filter (C-FMSB) that uses a suboptimum approach combining two criteria, the Fisher criterion (FC) and the SNR maximization (see details in [26]). Considering the averaged epochs $E_{N \times L}$, where $N=12$ is the number of electrodes and

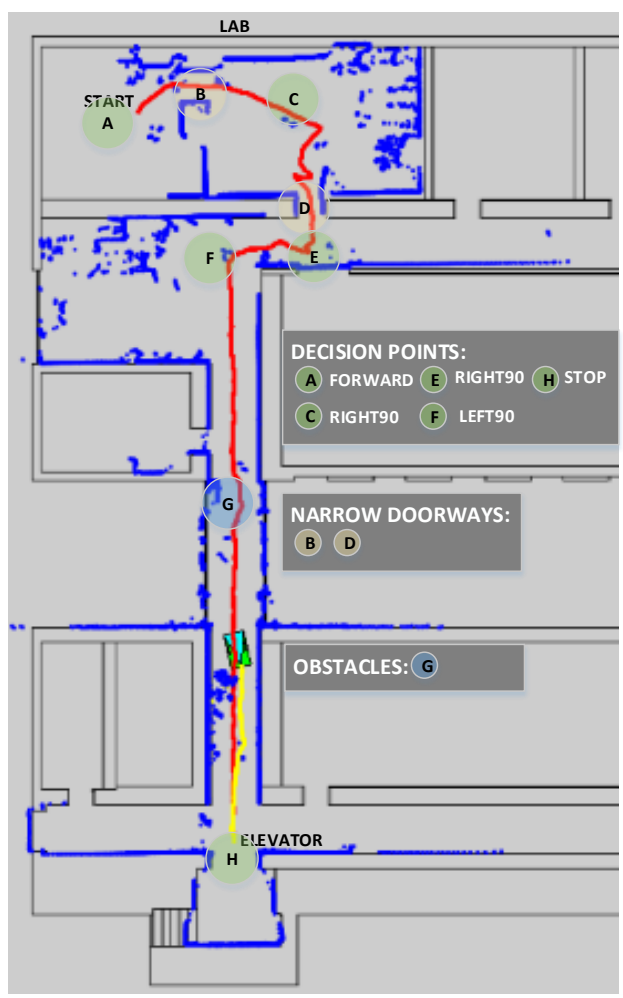

Fig. 3: Map with scenarios in which the participants performed Task2 with one obstacle (G), 2 narrow passages (B and D) and 5 decision points (A, C, E, F and $\mathrm{H}$ ).

$L=256$ is the number of samples, the spatial filter projection is obtained from

$$
Z_{1: 2}=W_{1: 2}^{T} E
$$

where $W_{1: 2}$ are the 2 optimal filters that correspond to the eigenvectors associated with the largest eigenvalues obtained from the solution of the generalized eigenvalue decomposition using both FC criteria and Max-SNR. The resulting feature vector is the concatenation of the two projections, $V_{1 \times 2 T}=$ $\left[\begin{array}{ll}z_{1} & z_{2}\end{array}\right]$, corresponding to 512 features. From these, the 120 most relevant features are selected using the R-square correlation method, leading to a feature vector $F_{1 \times 120}$. The feature 


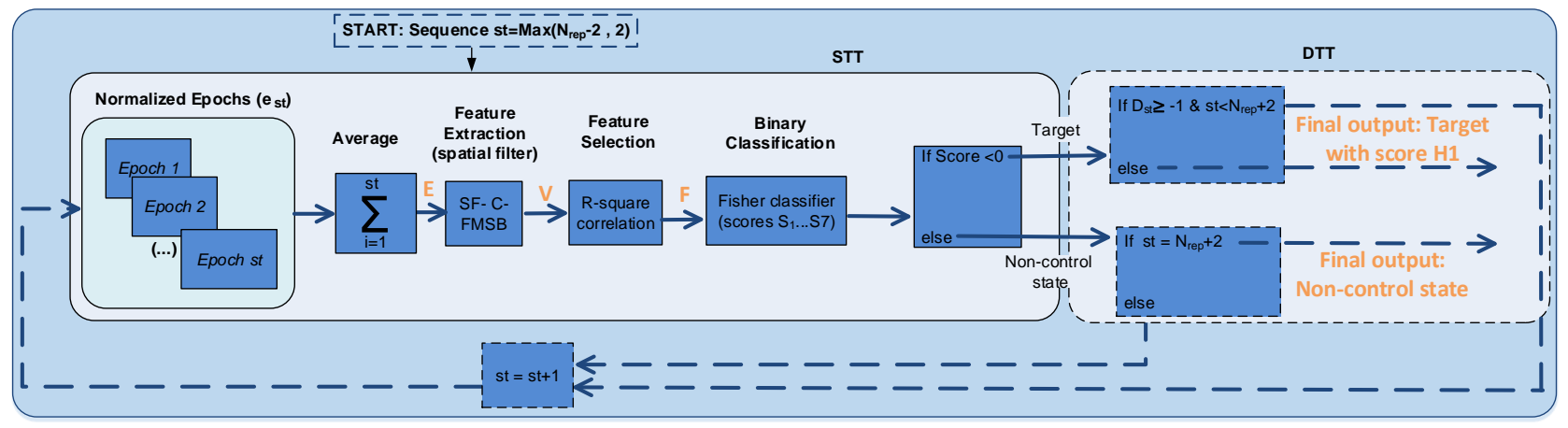

Fig. 4: Schematic representation of the overall pipeline of the self-paced P300-based BCI. Symbols E, V, F, and H1 refer to the averaged epochs, extracted features, selected features, and symbol with the highest score, respectively. Main solid line block represents the self-paced approach with STT. The dashed line blocks complement the STT block to implement the DTT approach (according to Algorithm 1).

vector is then classified by a Fisher Linear Discriminant (FLD) classifier, as described in sections III-B and III-C.

\section{B. Self-Paced Mode}

In self-paced mode, the BCI system needs to detect control and non-control states. The user can initiate his/her intention to enter the idle (non-control) state asynchronously whenever he/she wants, however, the system detection is made only at the end of each slot time (trial time). In the control state, participants were asked to focus on the target symbols, whilst in the non-control state (user has no intention of selecting a target), participants were asked to keep looking at the screen, but being relaxed without specifically attending to any of the symbols. This was thought as the most realistic scenario in those situations where the $\mathrm{BCI}$ is controlled by users unable to perform any motor movement. This scenario is different from most of the proposed P300-based BCWs that require a mental task to switch to the non-control state, for example, closing the eyes, performing mental tasks (e.g., reading a newspaper), selecting an extra symbol, or combining different neural mechanisms (e.g. P300 with motor imagery) [14], [15], [16], [17], [21]. The self-paced mode comprises 3 classes: target, non-target, and non-control state. Preliminary analyses, during which the calibration was performed with these three classes, showed that the epochs from the non-control class are very similar to non-target epochs, producing a similar classification score distribution as shown in the histogram of Fig. 5. Thus, the 3-class classification problem could be then transformed into a binary classification, and it was not necessary to consider the non-control state epochs to detect this class, nor to collect these epochs during calibration (we only need to collect target and non-target epochs). The Fisher classifier scores are positive for non-target and non-control epochs and negative for target epochs (the boundary is set to 0 ). However, the values of the scores depend on the threshold $\alpha$, set for each participant, which adjusts the false positive rate as explained ahead, corresponding to virtually move the decision boundary (i.e., instead of moving the boundary, the scores are moved left or right according to the threshold).

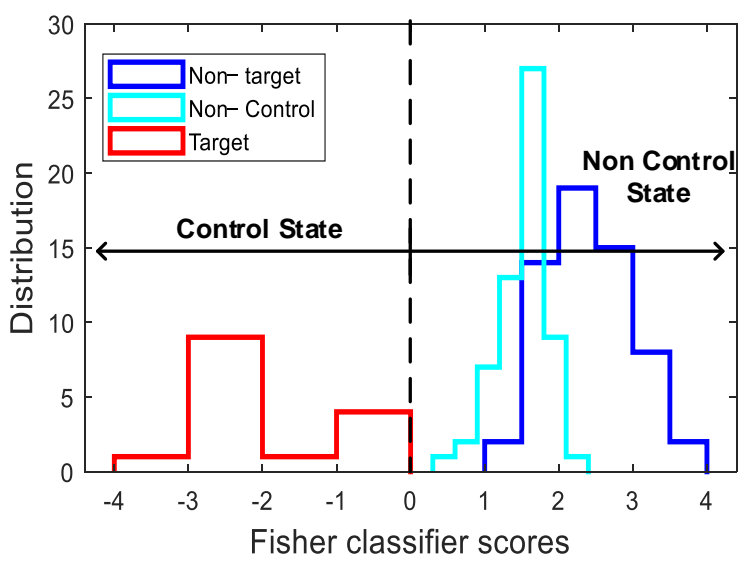

Fig. 5: Distribution (histogram) of FLD classifier scores of target, non-target, and non-control epochs obtained from a representative participant.

Considering the most discriminative features $F_{i}$ from each class, where $i \in\{+,-\}$ (target (+) and non-target (-)), the FLD projection is obtained as

$$
\mathbf{y}_{\mathbf{i}}=w \mathbf{F}_{\mathbf{i}}+\mathbf{b}
$$

where $w$ is the linear discriminative vector, and $b$ is defined as

$$
\mathrm{b}=-\frac{\left(b_{1}+b_{2}\right)}{2}
$$

with $b_{1}$ and $b_{2}$ computed from

$$
\begin{gathered}
\mathbf{b}_{1}=\frac{1}{K_{+}} \sum_{k=1}^{K_{+}} \mathbf{Y}_{+}+\sigma\left(\mathbf{Y}_{+}\right) \\
\mathbf{b}_{2}=\frac{1}{K_{-}} \sum_{k=1}^{K_{-}} \mathbf{Y}_{-}-\alpha \times \sigma\left(\mathbf{Y}_{-}\right)
\end{gathered}
$$

where $K_{i}$ is the number of training samples in class $i, \sigma$ is the standard deviation of target events and non-control state and $\alpha$ is a threshold that adjusts the false positive rate $(F P R)$. False Positives (FP) occur when the user does not intend to 
convey a command (non-control state) but the system detects a command. False Negatives (FN) occur when the user wants to provide a command but the systems detects a non-control state. The classifier is tuned to minimize the false positive rate $(F P R)$ as it is considered that the impact of a false noncontrol state is better than a wrong command. FPs can lead to unwanted trajectories of the wheelchair which may render complicated to return to the desired destination goal (e.g., a unwanted 'BACK' command). Yet, when passing through decision points, FNs can also lead to unwanted navigation paths, but as the the wheelchair speed slows down at decisions points, the user has more than one chance to provide the desired command. The threshold $\alpha$ was set experimentally for each participant before the experiment, with increments of 0.25 within the interval $[1: 0.25: 3]$. Using the calibration data, the FPR and FNR are computed for each increment of $\alpha$, and then the $\alpha$ that produces the lowest FPR and an FNR less than $10 \%$ is selected.

\section{Dynamic Trial Approach}

The DTT approach adjusts dynamically the number of repetitions to user's performance, balancing BCI speed and performance. Throughout the online operation, the P300 classification is computed for each sub-trial of index st. This value varies between $N_{r e p}-2$ and $N_{r e p}+2$ and its minimum value is limited to 2. $N_{\text {rep }}$ is set in the calibration session matching a $90 \%$ offline classification accuracy $\left(N_{r e p}\right.$ is the same for STT and DTT). The overall DTT approach is described in Algorithm 1 and schematically represented in Fig. 4. Starting with $s t=\operatorname{Max}\left(N_{\text {rep }}-2,2\right)$, the EEG signal is segmented into epochs, pre-processed and averaged. Features are then extracted, selected and classified using the models trained in the calibration session. A decision parameter $D_{s t}$, that defines the desired degree of confidence to recognize the target command, is computed as the normalized difference between the symbol with the highest score $\left(H_{1}\right)$ and second-highest score $\left(H_{2}\right)$. If the $D_{s t}$ value is less than -1 (an empirical value set experimentally and the same for all participants), a valid prediction is identified, the classification output is the target with score $H_{1}$, and the system proceeds to the next detection. Otherwise, there is a null prediction, that is, no target symbol is identified during the sequence $s t$ and the vector with epoch $\left(E_{s t}\right)$ is updated by adding the next epoch $(s t=s t+1)$. This procedure is repeated until a valid prediction is obtained or the number of sequences is equal to $N_{r e p}+2$.

\section{Online metrics}

We evaluate the feasibility of the BCI system through the accuracy $\left(A c c_{B C I}\right)$, number of $F P$ and $F N$ (see definition in section III-B), and the number of wrong detected targets $(W T)$. A WT occurs when the BCI detects correctly a controlstate (the user is willing to send a command) but the selected target command is wrong (e.g., the user wants to issue a 'FORWARD' command and the BCI detects 'LEFT'. The BCI accuracy is defined as

$$
\operatorname{Acc}_{B C I}=\frac{\text { Total }_{\text {com }}-(F P+F N+W T)}{\text { Total }_{\text {com }}}
$$

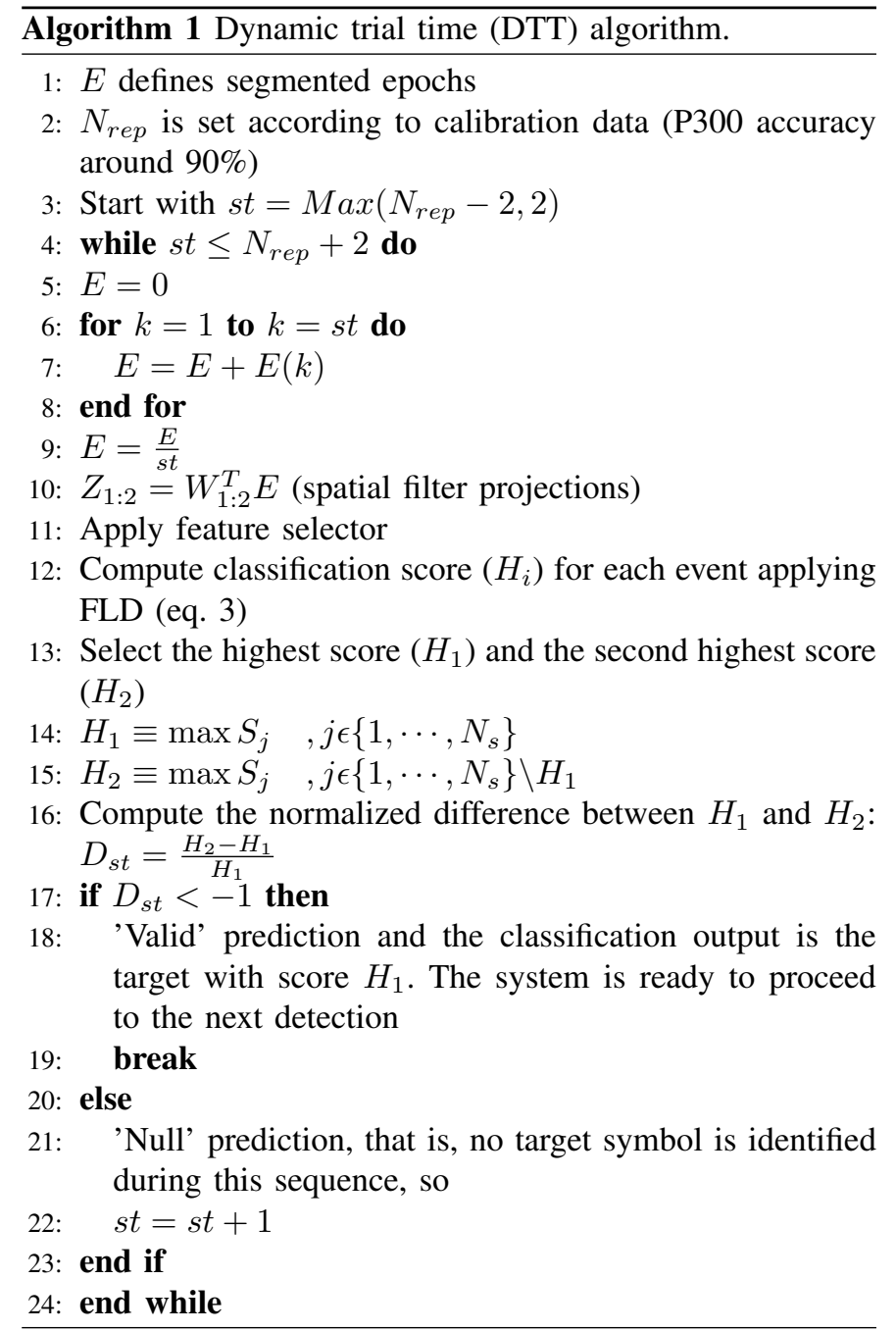

where Total ${ }_{\text {com }}$ is the total number of selections, i.e., the sum of the number of control commands $(C C)$, i.e., target selections, and the number of non-control commands $(N C C)$, i.e., trials in which the user does not want to select any target.

The global accuracy of the $\mathrm{BCW}$ is referred to as $A c c_{B C W}$ and was computed taking into account the performance of the collaborative controller:

$$
A c c_{B C W}=1-\frac{B C W_{e r r}}{C C+N C C}
$$

where $B C W_{e r r}$ is the number of the overall $\mathrm{BCW}$ errors, that is, the number of wrong commands at the output of the collaborative controller.

\section{EXPERIMENTAL Results}

The experiments were conducted with 7 able-bodied participants $(S 1$ to $S 7$ ) and 6 physically disabled participants ( $P 1$ to $P 6$ ) described in Tables I and II. Participants of Group I performed the three navigation tasks, namely, Task 1 , Task 2 , Task3, and participants of Group II performed only Task 1 and Task 2 . In Task 1 , it was used the self-paced control with STT approach (fixed number of repetitions, $N_{\text {rep }}$ ). In Task2, it was used the self-paced control with the DTT approach (number of repetitions was automatically adjusted 
online to user's performance). The navigation time (from starting point to the final destination) took on average 11 and 8 minutes respectively for Task 1 and Task 2 . Task 3 ran in nonself-paced mode, taking on average 11 minutes. The overall experiment lasted between 2 hours and a half and 3 hours for the group I, and between 3 hours and 3 hours and a half for the group II, including setup, calibration, familiarization, navigation times, and questionnaires. Disabled participants did not perform Task 3 because the time allowed by their institution to carry out the experiments was not enough to accomplish all three tasks. On the other hand, the results of Task3 obtained with Group I were very conclusive about the high difficulty and workload in using a non-self paced approach, so it was considered that this task would be unnecessary and unsuitable for the disabled participants. It should be stressed out that participants coming from APCC suffer from severe motor disabilities and required complicated transportation logistics to travel to the site of the experiments. Additionally, it was required that each participant was accompanied by a therapist or caregiver and a psychologist during the whole experimental process.

\section{A. BCI performance}

All commands received by the Hybrid Motion Planner of the RobChair result from the combination of the detected BCI command with the collaborative controller. Therefore, we need to assess both BCI accuracy $\left(A c c_{B C I}\right)$ and "BCI + collaborative controller" accuracy $\left(A c c_{B C W}\right)$ to analyze the impact of each module. The online results obtained for Task1, Task 2 and Task 3 are presented in Table III, Table IV and Table V. For Group I, the average BCI classification accuracies, calculated according to (7), are $97.1 \%, 94.5 \%$ and $89.1 \%$ for Task 1 , Task 2 and Task3, respectively. The number of commands provided by the users has been also calculated as it is one of the most important quantitative metrics to assess user effort and continuous workload. To accomplish Task1, participants in Group I needed to issue on average 10 control commands (target selections), while in Task3 the same group issued on average 73 control commands (same path of Task 1 ). Although in Task 1 only 5 decisions were necessary to reach the final destination, participants provided 5 extra commands on average due to wrong $\mathrm{BCW}$ commands or due to localization problems. For example, sometimes RobChair missidentified local deadlocks stopping, thereby requiring new commands from the user that led to a trajectory replanning. The self-paced mode used in Task 1 has clearly shown its effectiveness in considerably decreasing the number of control commands required to drive the RobChair. It is also possible to conclude that participants spent on average $86.3 \%$ of the time in a state of non-control, which undoubtedly greatly reduced the time that users were focused on target selection, with an expected positive impact on users' workload. The collaborative controller increased the overall accuracy of Task1, Task 2 and Task 3 by $2.9 \%, 4.5 \%$ and $5.5 \%$ respectively, reaching $100 \%, 99.1 \%$ and $94.6 \%$, leading to a very high reliability of the overall system. None of the BCI errors made by Group I in Task 1 had an impact on the navigation as the collaborative controller rejected them all. For Group II, BCI results were just slightly lower than for Group I, but the BCW accuracy was almost the same, as the collaborative controller corrected most of wrong BCI commands. These results show the effectiveness of the "Self-paced + collaborative" control approach, with performance remaining stable across patients with varied levels of physical disability.

TABLE III: Online performance for both groups in Task1: self-paced mode with static trial time

\begin{tabular}{llllllllll}
\hline Subjects & CC & NCC & WT & FP & FN & $\begin{array}{l}\text { BCW } \\
\text { err }\end{array}$ & TT & $\begin{array}{l}\operatorname{Acc}_{B C I} \\
(\%)\end{array}$ & $\begin{array}{l}\operatorname{Acc}_{B C W} \\
(\%)\end{array}$ \\
\hline S1 & 8 & 61 & 0 & 1 & 2 & 0 & 7.1 & 95.7 & 100.0 \\
S2 & 12 & 72 & 0 & 0 & 2 & 0 & 5.9 & 97.6 & 100.0 \\
S3 & 11 & 58 & 0 & 0 & 0 & 0 & 7.1 & 100.0 & 100.0 \\
S4 & 10 & 78 & 0 & 0 & 0 & 0 & 7.1 & 100.0 & 100.0 \\
S5 & 10 & 66 & 0 & 0 & 2 & 0 & 7.1 & 97.4 & 100.0 \\
S6 & 7 & 57 & 0 & 0 & 2 & 0 & 8.4 & 96.9 & 100.0 \\
S7 & 14 & 49 & 1 & 4 & 0 & 0 & 7.1 & 92.1 & 100.0 \\
Average & $\mathbf{1 0 . 3}$ & $\mathbf{6 3 . 0}$ & $\mathbf{0 . 1}$ & $\mathbf{0 . 7}$ & $\mathbf{1 . 1}$ & $\mathbf{0 . 0}$ & $\mathbf{7 . 1}$ & $\mathbf{9 7 . 1}$ & $\mathbf{1 0 0 . 0}$ \\
\hline P1 & 14 & 63 & 1 & 3 & 2 & 1 & 7.1 & 92.2 & 98.7 \\
P2 & 23 & 104 & 1 & 5 & 1 & 0 & 7.1 & 94.5 & 100.0 \\
P3 & 13 & 85 & 0 & 2 & 4 & 0 & 7.1 & 93.9 & 100.0 \\
P4 & 13 & 68 & 0 & 3 & 4 & 1 & 7.1 & 91.4 & 98.8 \\
P5 & 17 & 77 & 0 & 0 & 1 & 0 & 5.9 & 98.9 & 100.0 \\
P6 & 12 & 66 & 0 & 2 & 0 & 0 & 7.1 & 97.4 & 100.0 \\
Average & $\mathbf{1 5 . 3}$ & $\mathbf{7 7 . 2}$ & $\mathbf{0 . 3}$ & $\mathbf{2 . 5}$ & $\mathbf{2 . 0}$ & $\mathbf{0 3}$ & $\mathbf{6 . 9}$ & $\mathbf{9 4 . 7}$ & $\mathbf{9 9 . 6}$ \\
\hline
\end{tabular}

$\mathrm{CC}=$ number of control commands, $\mathrm{NCC}=$ number of non-control commands, WT $=$ wrong targets, $\mathrm{FP}=$ False Positives, $\mathrm{FN}=$ False Negatives, $\mathrm{BCW}_{\text {err }}=$ number the $\mathrm{BCW}$ errors, $\mathrm{TT}=$ overall trial time, $\operatorname{Acc}_{B C I}$ is the BCI accuracy, $\operatorname{Acc}_{B C W}$ is the BCW accuracy.

The average number of FP and FN shown in Tables III and IV are respectively 1.1 and 0.7 for Group I, and 1.9 and 2.0 for Group II, showing that the control vs. non-control state detection is very effective. The comparison between the BCI accuracy obtained for Task 1 and Task 2 gives a measure of the impact of the DTT approach. The BCI classification accuracy was high but lower than using the STT (Task1), and the time to select a command was reduced in about $1 \mathrm{~s}$ for both groups (paired t-test, $p=0.003$ and $p=0.03$ ). This shows that the dynamic trial time can be used to adjust the BCI speed vs. accuracy. In order to favor accuracy, the DTT method should be more restrictive in the degree of confidence of the command (given by $D_{s t}$ in Algorithm 1).

\section{B. Subjective questionnaires}

Participants were asked to answer two questionnaires assessing their subjective perception of the performed tasks. The first questionnaire was based on the NASA-TLX [27] to assess mental demand, physical demand, temporal demand, performance, effort and frustration of the three tasks. Only one part of the NASA-TLX has been applied, that is, the participants rated each subscale but did not evaluate the contribution of each factor (weight). The overall workload for each subject is therefore an unweighted average of these six subscales. The second questionnaire was a customized questionnaire that compared the 3 tasks regarding the degree of user satisfaction, with questions directed to the specific tasks. The NASA-TLX workload scores range between 0 and 100 (21 graduations), while the customized questionnaire ranged 
TABLE IV: Online performance for both groups in Task2: self-paced mode with dynamic trial time

\begin{tabular}{llllllllllll}
\hline Subjects & CC & NCC & WT & FP & FN & $\mathrm{BCW}_{\text {err }}$ & $\mathrm{TT}_{\text {Max }}$ & $\mathrm{TT}_{\text {Min }}$ & $\mathrm{TT}_{\text {Mean }}$ & $\begin{array}{l}\mathrm{Acc}_{B C I} \\
(\%)\end{array}$ & $\begin{array}{l}\mathrm{Acc}_{B C W} \\
(\%)\end{array}$ \\
\hline S1 & 7 & 27 & 0 & 1 & 0 & 1 & 5.9 & 4.7 & 5.5 & 97.1 & 97.1 \\
$\mathrm{~S} 2$ & 11 & 31 & 0 & 0 & 0 & 0 & 8.4 & 3.5 & 4.9 & 100.0 & 100.0 \\
S3 & 14 & 27 & 0 & 0 & 0 & 0 & 9.6 & 5.9 & 6.7 & 100.0 & 100.0 \\
S4 & 10 & 31 & 0 & 1 & 0 & 0 & 5.9 & 4.7 & 5.8 & 97.6 & 100.0 \\
S5 & 19 & 30 & 0 & 4 & 1 & 0 & 9.6 & 5.9 & 6.4 & 89.8 & 100.0 \\
S6 & 10 & 23 & 0 & 1 & 1 & 0 & 10.8 & 5.9 & 8.0 & 93.9 & 100.0 \\
S7 & 41 & 23 & 7 & 4 & 0 & 2 & 8.4 & 4.7 & 6.3 & 82.8 & 96.9 \\
Average & $\mathbf{1 6 . 0}$ & $\mathbf{2 7 . 4}$ & $\mathbf{1 . 0}$ & $\mathbf{1 . 6}$ & $\mathbf{0 . 3}$ & $\mathbf{0 . 4}$ & $\mathbf{8 . 4}$ & $\mathbf{5 . 0}$ & $\mathbf{6 . 2}$ & $\mathbf{9 4 . 5}$ & $\mathbf{9 9 . 1}$ \\
\hline P1 & 9 & 33 & 0 & 0 & 2 & 0 & 7.1 & 7.1 & 7.1 & 95.2 & 100.0 \\
P2 & 36 & 36 & 5 & 6 & 2 & 1 & 9.6 & 4.7 & 6.8 & 81.9 & 98.6 \\
P3 & 9 & 31 & 0 & 0 & 1 & 0 & 9.6 & 5.9 & 7.1 & 97.5 & 100.0 \\
P4 & 12 & 79 & 0 & 1 & 4 & 1 & 5.9 & 4.7 & 5.7 & 94.5 & 98.9 \\
P5 & 13 & 50 & 0 & 0 & 0 & 0 & 4.7 & 3.5 & 4.4 & 100.0 & 100.0 \\
P6 & 8 & 31 & 1 & 1 & 3 & 0 & 7.4 & 5.2 & 6.2 & 87.2 & 100.0 \\
Average & $\mathbf{1 4 . 5}$ & $\mathbf{4 3 . 3}$ & $\mathbf{1 . 0}$ & $\mathbf{1 . 3}$ & $\mathbf{2 . 0}$ & $\mathbf{0 . 3}$ & $\mathbf{7 . 4}$ & $\mathbf{5 . 2}$ & $\mathbf{6 . 2}$ & $\mathbf{9 2 . 7}$ & $\mathbf{9 9 . 6}$ \\
\hline
\end{tabular}

$\mathrm{CC}=$ number of control commands, $\mathrm{NCC}=$ number of non-control commands, WT $=$ wrong targets, $\mathrm{FP}=$ False Positives, $\mathrm{FN}=$ False Negatives, $\mathrm{BCW}_{\text {err }}=$ number of $\mathrm{BCW}$ errors, $\mathrm{TT}_{\text {Max }}$ $=$ maximum trial time, $\mathrm{TT}_{M i n}=$ minimum overall trial time, $\mathrm{TT}_{\text {Mean }}=$ mean of trial time, $\operatorname{Acc}_{B C I}$ is the BCI accuracy, $\operatorname{Acc}_{B C W}$ is the BCW accuracy.
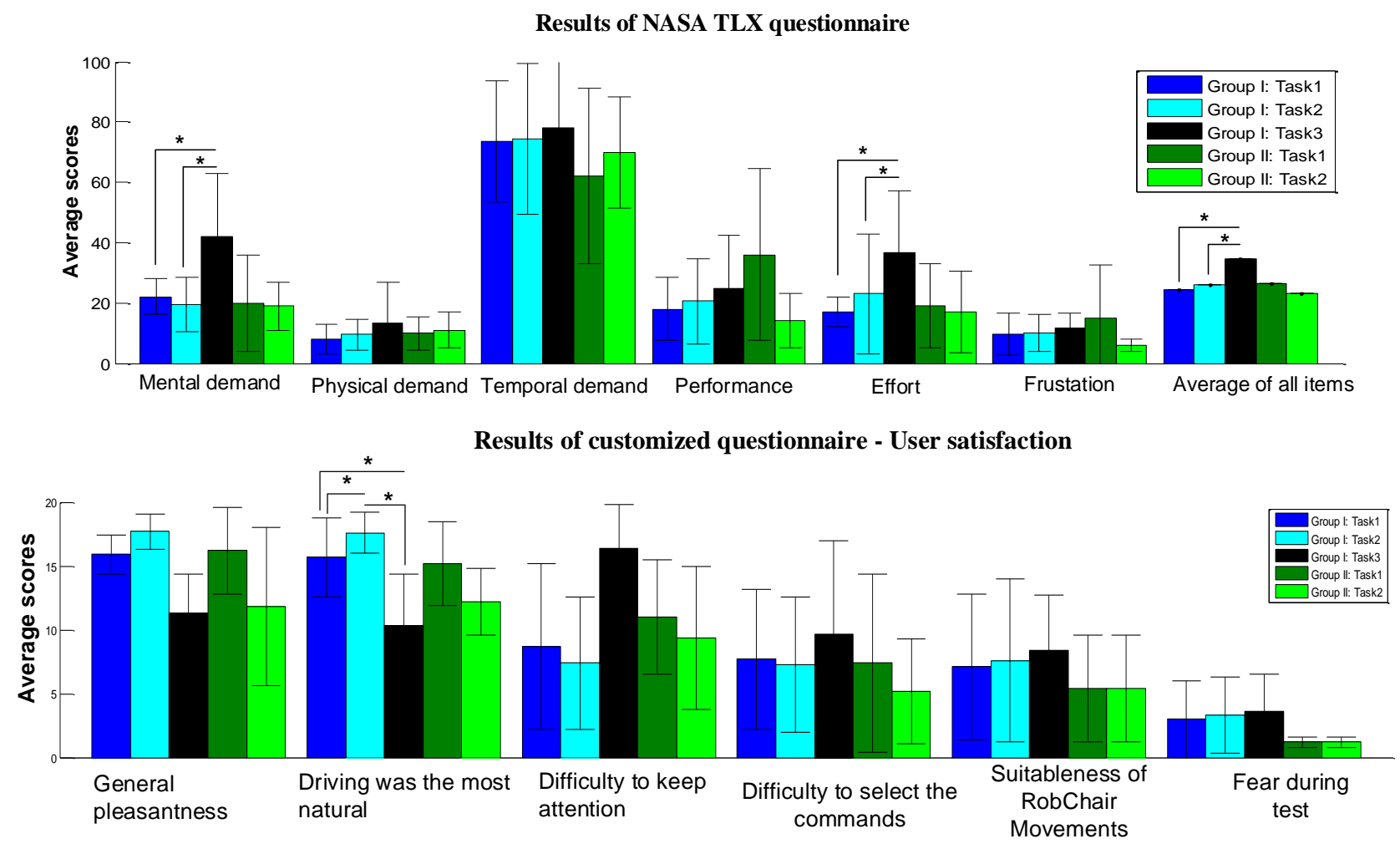

Fig. 6: Results of the questionnaires for each group. Top: NASA TLX raw rating scores and unweighted average of all items (scale 0-100); and Bottom: results of user-satisfaction customized questionnaire (scale 1-20). $(*)$ indicates items that were statistically significant.

from 1 (very low) to 20 (very high). For the performance parameter, the scale is inverted, i.e., 1 is perfect and 20 is failure. In addition, psychometric questionnaires were applied to Group II to assess the emotional state according to StateTrait Anxiety Inventory (STAI) [28] and the Beck Depression Inventory (BDI) [29], in order to ensure that the participants were emotionally capable of performing the tasks.
The average results of the two subjective questionnaires are in Fig. 6. Group I reported mental demand and effort as significantly higher in Task3, compared to Task1 (diff=20.0, paired t-test, $\mathrm{p}=0.01$, and $\operatorname{diff}=19.5, \mathrm{p}=0.02$, respectively), and compared to Task2 (diff $=22.5$, paired t-test, $\mathrm{p}=0.006$, and diff $=13.5, \mathrm{p}=0.002$, respectively), as expected from the quantitative results. Task2 was scored as slightly less mental 
TABLE V: Online Performance for healthy participants (Group I) in Task3: non self-paced mode with static trial-time

\begin{tabular}{lccccll}
\hline Subjects & CC & WT & $\mathrm{BCW}_{\text {err }}$ & TT & $\begin{array}{l}\operatorname{Acc}_{B C I}\left(\begin{array}{l}\mathrm{Acc}_{B C W} \\
(\%)\end{array}\right. \\
(\%)\end{array}$ \\
\hline S1 & 85 & 21 & 10 & 7.1 & 75.3 & 88.2 \\
S2 & 73 & 2 & 2 & 5.9 & 97.3 & 97.3 \\
S3 & 65 & 4 & 4 & 7.1 & 93.8 & 93.8 \\
S4 & 71 & 4 & 4 & 7.1 & 94.4 & 94.4 \\
S5 & 85 & 7 & 3 & 7.1 & 91.8 & 96.5 \\
S6 & 62 & 11 & 3 & 8.4 & 82.3 & 95.2 \\
S7 & 71 & 8 & 2 & 7.1 & 88.7 & 97.2 \\
Average & $\mathbf{7 3 . 1}$ & $\mathbf{8 . 1}$ & $\mathbf{4 . 0}$ & $\mathbf{7 . 1}$ & $\mathbf{8 9 . 1}$ & $\mathbf{9 4 . 6}$ \\
\hline
\end{tabular}

$\mathrm{CC}=$ number of control commands, $\mathrm{NCC}=$ number of noncontrol commands, $\mathrm{WT}=$ wrong targets, $\mathrm{TT}=$ overall trial time, $\operatorname{Acc}_{B C I}$ is the BCI accuracy, $\operatorname{Acc}_{B C W}$ is the BCW accuracy.

demanding than Task 1 for both groups (diff=2.5 and diff=1), but the difference is not statistically significant. The perceived performance of Group I in Task1 was slightly greater than in Task 2 and Task3, although BCI classification accuracies of the 3 tasks were very similar. Group I classified Task3 as more temporal demanding, without significant differences between tasks (paired t-test, $p=0.26$ and $p=0.35$ ). For Group I, temporal demand was similar for Task 1 and Task2 (diff $=1)$, and for Group II Task2 was considered slightly less demanding $($ diff $=8)$. The scores for physical demand and frustration of both groups are low (less than 3). These results suggest that participants felt comfortable and fearless. The average of Group I for NASA-TLX items shows that Task 3 was the task with the highest taskload (paired t-test, $p=0.03$ and $p=0.009$ ). For Group II, Task 2 presents the lower taskload, but the difference is not statistically significant. Comparative results between Task 1 and Task 2 could have been influenced by the difference between tasks. Although both tasks have the same number of decision points, Task2 has a shorter path and fewer obstacles (still, the participants issued more CC in Taks2).

In the second questionnaire, Task 3 was reported as the less pleasant, less natural, more difficult to keep attention and the more difficult to select commands, which again corroborates the results of the quantitative metrics. Participants of Group I classified Task 2 as more natural than Task1 $(p=0.03)$. The difference in pleasantness between Task 1 and Task 2 was not statistically significant for both groups. Regarding the perception of "Suitableness of RobChair Movements", Group II considered the movements more appropriate than Group I.

\section{DISCUSSION}

A brain-controlled wheelchair is a complex system that requires a high level of reliability and safety and involves intelligent navigation systems. The goal of this study was to assess the impact of the combination of a collaborative controller with a self-paced control (using STT and DTT approaches) on users' effort, naturalness of interaction and system reliability when driving a robotic wheelchair with a BCI. Able-bodied and motor impaired participants used the proposed self-paced BCI with a mean accuracy of $95.8 \%$ and $93.7 \%$, respectively. These results show the effectiveness of the
BCI classifier and in particular of the control vs. non-control state detection. Still, the average number of FP was higher than the number of FN, which was not what was intended. A posterior offline analysis made after the experiments showed that a better tuning of the threshold $\alpha$ could have decreased the number of false positives. The collaborative controller increased the overall system accuracy to above $99 \%$ for both groups, clearly showing its importance for the reliability of the BCW. Even using the non self-paced approach (which yielded a $89 \%$ BCI accuracy) the collaborative controller increased the overall $\mathrm{BCW}$ accuracy to $94.6 \%$. The collaborative controller proved to have the desired effect, by discarding wrong BCI commands and replacing them by the intended ones, thereby reducing the impact of lower BCI performances. The self-paced control enormously reduced the number of the required commands, specifically from 73 to 10 on average. This reduction had a significant impact on the perceived overall task workload as shown in Fig. 6, in particular on mental demand and effort. Accordingly, the greater workload of the non self-paced operation was reflected in a decrease of the BCI accuracy in $8.0 \%$, when compared to the self-paced operation. Analysing Task 1 vs. Task 2 it was found that the DTT increased the BCI speed by reducing the time per trial in about $1 \mathrm{sec}$, but it slightly decreased the BCI performance, which was not the desired outcome. Based on these results, we can state that the self-paced approach had a very high impact on the reliability, naturalness, and workload demand of the $\mathrm{BCW}$, and the collaborative controller had a high impact on the reliability of the $\mathrm{BCW}$, with increased relevance when the BCI performance was lower. Although with a lower impact on the entire system, the DTT approach showed the possibility of adjusting BCI speed vs. user's performance. This will be a subject of future research. For example, the $D_{s t}$ threshold should be individually tuned for each participant to ensure an improvement of the BCI accuracy. Moreover, the impact of the DTT approach may have been diminished by the high positive impact of the self-paced control, since the user is less susceptible to lack of attention and fatigue. Overall, participants scored Task 1 and Task 2 very similarly. As regards participants' subjective preference in performing the designated tasks, there were no significant differences between the two groups. The subjective results show a very positive user experience feedback regarding workload demand and naturalness of control of the overall system.

Table VI shows a comparison between different brainactuated wheelchair architectures that are closely related to our system, i.e., that use a control scheme combining user and machine commands, a self-paced paradigm and experiments with real wheelchairs. Only one of the studies reported experiments with motor disabled participants [21], which emphasizes the need for more studies involving the potential target users, in a perspective of human-centred design. Additionally, most of the experiments of the proposed works were performed in highly structured environments set up in lab. Our work presents the most complex navigation scenario including both healthy and severely motor disabled participants. To the best of our knowledge our proposal is the only one achieving an overall accuracy greater than $99 \%$, which validates the 
TABLE VI: Summary of related BCW works that use a collaborative control, a self-paced paradigm and experiments with real wheelchair.

\begin{tabular}{|c|c|c|c|c|}
\hline Study & BCI approach & Self-paced paradigm & Environment & Subjects \\
\hline Rebsamen et al. [14] & $\mathrm{P} 300$ and $\mathrm{MI}$ & Static trial & $\begin{array}{l}\text { Structured environment based on corridors and } \\
\text { rooms without obstacles. }\end{array}$ & Healthy: 5 \\
\hline $\begin{array}{l}\text { Carlson and Millan } \\
{[5]}\end{array}$ & MI & Static trial & $\begin{array}{l}\text { Unstructured environment based on an office room } \\
\text { with static obstacles. }\end{array}$ & Healthy: 4 \\
\hline Wang al. [15] & $\begin{array}{l}\text { MI and P300 } \\
\text { and blink }\end{array}$ & Static trial & $\begin{array}{l}\text { Semi-structured environment based on corridors with } \\
\text { static obstacles. }\end{array}$ & Healthy: 4 \\
\hline Zhang al. [16] & MI or P300 & Static trial & $\begin{array}{l}\text { Semi-structured environment based on a domestic } \\
\text { room with static obstacles. }\end{array}$ & Healthy: 9 \\
\hline He al. [21] & P300 & Static trial & Real. Trajectory with two destinations in a room. & $\begin{array}{l}\text { Healthy: } 8 ; \\
\text { Disabled: } 5\end{array}$ \\
\hline Our study & P300 & $\begin{array}{l}\text { Static and Dynamic } \\
\text { trial }\end{array}$ & $\begin{array}{l}\text { Semi-structured environment based on office } \\
\text { rooms and corridors with static obstacles and } \\
\text { narrow passages. }\end{array}$ & $\begin{array}{l}\text { Healthy: } 7 \\
\text { Disabled: } 6\end{array}$ \\
\hline
\end{tabular}

proposed BCI and navigation approaches. Moreover, from this group of studies our study is the only one assessing user experience through subjective questionnaires.

Users steered the wheelchair in office-like environments requiring challenging tasks, such as narrow door passages and obstacle (static and dynamic) avoidance. Although complex, the scenarios were still very controlled and different from users' daily home settings. The experimental procedures were also very controlled, as the research team was always assisting the tasks. The good results achieved by motor disabled participants suggests that the proposed BCI may represent an effective solution for wheelchair control. The overall results have been very promising and motivate new further research already under way, namely the integration of vision sensors to recognize semantic features, such as doors, tables, chairs, which will be incorporated dynamically as target goals in the interface. Error- related potentials (ErrP) that we have already used in a different context [30] are also being integrated to improve the reliability of the BCI commands.

\section{CONCLUSION}

This study assessed the impact of the integration of collaborative control, self-paced control, and dynamic-time commands into a BCW system. The system was validated by 7 healthy participants and 6 motor disabled patients in real office-environment navigation tasks. Both able-bodied and motor disabled participants successfully controlled the BCI system with an average BCI accuracy of $95.8 \%$ and $93.7 \%$ respectively and the collaborative controller corrected most of wrong commands increasing the accuracy to more than $99 \%$ for both groups. The subjective results corroborate the quantitative results, showing a positive impact of self-paced and collaborative control. These results are promising for the effective and tailored use of BCWs by individuals with severe motor impairments. Still, more extensive experiments with a wider group of participants and in more natural living contexts are needed to validate the approaches.

\section{ACKNOWLEDGMENT}

Authors would like to thank all participants and staff of the Cerebral Palsy Association of Coimbra (APCC).

\section{REFERENCES}

[1] A. Davies, L. D. Souza, and A. Frank, "Changes in the quality of life in severely disabled people following provision of powered indoor/outdoor chairs," Disability and Rehabilitation, vol. 25, no. 6, pp. 286-290, 2003.

[2] A. Frank, J. Ward, N. Orwell, C. McCullagh, and M. Belcher, "Introduction of a new NHS electric-powered indoor/outdoor chair (epioc) service: benefits, risks and implications for prescribers," Clinical Rehab., vol. 14, no. 6, pp. 665-673, 2000.

[3] J. R. Wolpaw, N. Birbaumer, D. J. McFarland, G. Pfurtscheller, and T. M. Vaughan, "Brain-computer interfaces for communication and control," Clinical neurophysiology, vol. 113, no. 6, pp. 767-791, 2002.

[4] A. C. Lopes, G. Pires, and U. Nunes, "Assisted navigation for a brainactuated intelligent wheelchair," Robotics and Autonomous Systems, vol. 61 , no. 3, pp. $245-258,2013$.

[5] T. Carlson and J. d. R. Millan, "Brain-controlled wheelchairs: a robotic architecture," IEEE Robotics \& Automation Magazine, vol. 20, no. 1, pp. $65-73,2013$.

[6] A. Lopes, J. Rodrigues, J. Perdigao, G. Pires, and U. Nunes, "A new hybrid motion planner: Applied in a brain-actuated robotic wheelchair," IEEE Robotics \& Automation Magazine, vol. 23, no. 4, pp. 82-93, 2016.

[7] A. Kübler, E. M. Holz, E. W. Sellers, and T. M. Vaughan, "Toward independent home use of brain-computer interfaces: a decision algorithm for selection of potential end-users," Archives of physical medicine and rehabilitation, vol. 96, no. 3, pp. S27-S32, 2015.

[8] L. Bi, X. Fan, and Y. Liu, "EEG-based brain-controlled mobile robots: A survey," IEEE Transactions on Human-Machine Systems, vol. 43, no. 2, pp. 161-176, March 2013.

[9] T. Carlson and Y. Demiris, "Collaborative control for a robotic wheelchair: evaluation of performance, attention, and workload," IEEE Transactions on Systems, Man, and Cybernetics, Part B (Cybernetics), vol. 42, no. 3, pp. 876-888, 2012.

[10] J. Duan, Z. Li, C. Yang, and P. Xu, "Shared control of a brain-actuated intelligent wheelchair," in Proceeding of the 11th World Congress on Intelligent Control and Automation. IEEE, 2014, pp. 341-346.

[11] Z. Li, S. Zhao, J. Duan, C.-Y. Su, C. Yang, and X. Zhao, "Human cooperative wheelchair with brain-machine interaction based on shared control strategy," IEEE/ASME Transactions on Mechatronics, vol. 22, no. 1, pp. 185-195, 2016.

[12] I. Iturrate, J. M. Antelis, A. Kubler, and J. Minguez, "A noninvasive brain-actuated wheelchair based on a P300 neurophysiological protocol and automated navigation," IEEE transactions on robotics, vol. 25, no. 3, pp. 614-627, 2009.

[13] P. F. Diez, S. M. T. Müller, V. A. Mut, E. Laciar, E. Avila, T. F. BastosFilho, and M. Sarcinelli-Filho, "Commanding a robotic wheelchair with a high-frequency steady-state visual evoked potential based braincomputer interface," Medical engineering \& physics, vol. 35, no. 8, pp. $1155-1164,2013$.

[14] B. Rebsamen, C. Guan, H. Zhang, C. Wang, C. Teo, M. H. Ang, and E. Burdet, "A brain controlled wheelchair to navigate in familiar environments," IEEE Transactions on Neural Systems and Rehabilitation Engineering, vol. 18, no. 6, pp. 590-598, 2010.

[15] H. Wang, Y. Li, J. Long, T. Yu, and Z. Gu, "An asynchronous wheelchair control by hybrid EEG-EOG brain-computer interface," Cognitive neurodynamics, vol. 8, no. 5, pp. 399-409, 2014. 
[16] R. Zhang, Y. Li, Y. Yan, H. Zhang, S. Wu, T. Yu, and Z. Gu, "Control of a wheelchair in an indoor environment based on a braincomputer interface and automated navigation," IEEE transactions on neural systems and rehabilitation engineering, vol. 24, no. 1, pp. 128139,2015

[17] Y. Yu, Z. Zhou, Y. Liu, J. Jiang, E. Yin, N. Zhang, Z. Wang, Y. Liu, $\mathrm{X}$. Wu, and D. Hu, "Self-paced operation of a wheelchair based on a hybrid brain-computer interface combining motor imagery and p300 potential," IEEE Transactions on Neural Systems and Rehabilitation Engineering, vol. 25, no. 12, pp. 2516-2526, 2017.

[18] Y. Yu, Y. Liu, J. Jiang, E. Yin, Z. Zhou, and D. Hu, "An asynchronous control paradigm based on sequential motor imagery and its application in wheelchair navigation," IEEE Transactions on Neural Systems and Rehabilitation Engineering, vol. 26, no. 12, pp. 2367-2375, 2018.

[19] D. W.-K. Ng, Y.-W. Soh, and S.-Y. Goh, "Development of an autonomous BCI wheelchair," in 2014 IEEE Symposium on Computational Intelligence in Brain Computer Interfaces (CIBCI). IEEE, 2014, pp. $1-4$.

[20] T. Kaufmann, A. Herweg, and A. Kübler, "Toward brain-computer interface based wheelchair control utilizing tactually-evoked event-related potentials," Journal of neuroengineering and rehabilitation, vol. 11, no. 1, p. 7, 2014.

[21] S. He, R. Zhang, Q. Wang, Y. Chen, T. Yang, Z. Feng, Y. Zhang, M. Shao, and Y. Li, "A P300-based threshold-free brain switch and its application in wheelchair control," IEEE Transactions on Neural Systems and Rehabilitation Engineering, vol. 25, no. 6, pp. 715-725, 2016.

[22] K. Choi, "Control of a vehicle with EEG signals in real-time and system evaluation," European journal of applied physiology, vol. 112, no. 2, pp. 755-766, 2012.

[23] J. Tang, Y. Liu, D. Hu, and Z. Zhou, "Towards BCI-actuated smart wheelchair system," Biomedical engineering online, vol. 17, no. 1, p. 111,2018

[24] Á. Fernández-Rodríguez, F. Velasco-Álvarez, and R. Ron-Angevin, "Review of real brain-controlled wheelchairs," Journal of neural engineering, vol. 13, no. 6, p. 061001, 2016.

[25] S. Kohlbrecher, O. Von Stryk, J. Meyer, and U. Klingauf, "A flexible and scalable slam system with full 3d motion estimation," in 2011 IEEE International Symposium on Safety, Security, and Rescue Robotics. IEEE, 2011, pp. 155-160.

[26] G. Pires, U. Nunes, and M. Castelo-Branco, "Statistical spatial filtering for a P300-based BCI: tests in able-bodied, and patients with cerebral palsy and amyotrophic lateral sclerosis," Journal of neuroscience methods, vol. 195, no. 2, pp. 270-281, 2011.

[27] NASA, "Nasa task load index (tlx) v. 1.0 manual," 1986.

[28] C. Spielberg, R. Gorsuch, and R. Lushene, "Test manual for the statetrait anxiety inventory," 1970.

[29] A. T. Beck, C. H. Ward, M. Mendelson, J. Mock, and J. Erbaugh, "An inventory for measuring depression," Archives of general psychiatry, vol. 4, no. 6, pp. 561-571, 1961.

[30] A. Cruz, G. Pires, and U. J. Nunes, "Double ErrP Detection for Automatic Error Correction in an ERP-Based BCI Speller," IEEE Trans. on Neur. Syst. and Rehab. Eng., vol. 26, no. 1, pp. 26-36, 2018. 